\title{
Eficiencia de una terma solar con un colector de cobre y otro de CPVC
}

\section{Efficiency of a solar thermal with a copper collector and another of CPVC}

\author{
iD 'Miguel Ángel Barrena Gurbillón (iD) 2Godofredo Román Lobato Calderón, \\ iD 'Oscar Andrés Gamarra Torres iD 'Carla María Ordinola Ramírez
}

Universidad Nacional Toribio Rodríguez de Mendoza de Amazonas. Chachapoyas, Perú.

Universidad Católica Sedes Sapientiae. Lima, Perú. ${ }^{2}$

\section{Resumen}

Se determinó la eficiencia de una terma solar con un colector solar plano de tubos y accesorios de $0,0127 \mathrm{~m}\left(1 / 2{ }^{\prime \prime}\right)$ de policloruro de vinilo para agua caliente (CPVC) de 10 vueltas y otro similar de tubos y accesorios de cobre, cada uno de 1,5 m2 (1,5 m x $1 \mathrm{~m}$ y 0,1 m) conectados en paralelo; ubicados en dirección norte-sur, con lado sur levantado $30^{\circ}$, y tanque de almacenamiento de agua caliente de $120 \mathrm{~L}$ aislado térmicamente, en Tarma, Perú. Se consideró tres tipos de días: soleados (sol visible todo el día), combinados (presencia visible parcial del sol por nubosidad) y nublados (no hay presencia visible del sol); cada media hora desde las 8:00 hasta las 16:00 horas, se registró la radiación solar; temperatura ambiente y del interior de cada colector y temperatura del agua: que entra a los colectores solares, a la salida de cada colector y en la parte superior del tanque de almacenamiento. La eficiencia en día soleado fue $54,59 \%$, en día combinado $48,32 \%$ y en día nublado 30,01\%. Ganó más temperatura el colector de CPVC. Producirá agua más caliente una terma solar con dos colectores planos, con serpentines de 10 vueltas de tubería de CPVC de 1,5 m2 cada uno y tanque de almacenamiento de agua de $120 \mathrm{~L}$ aislado térmicamente.

Palabras clave: Eficiencia, Terma solar.

\section{Abstract}

The efficiency of a solar thermal was determined with a flat solar collector of pipes and accessories of $0.0127 \mathrm{~m}\left(1 / 22^{\prime \prime}\right)$ of polyvinyl chloride for hot water (CPVC) of 10 turns and another similar one of copper pipes and accessories, each one of $1.5 \mathrm{~m} 2(1.5 \mathrm{~m} \times 1 \mathrm{~m}$ and $0.1 \mathrm{~m})$ connected in parallel; Located in north-south direction, with south side raised $30^{\circ}$, and thermally insulated $120 \mathrm{~L}$ hot water

Recibido: Marzo 2020

Aceptado: Junio 2020

storage tank, in Tarma, Peru. Three types of days were considered: sunny (sun visible all day), combined (partial visible presence of the sun due to cloudiness) and cloudy (there is no visible presence of the sun); Every half hour from 8:00 to 16:00 hours, solar radiation was recorded; ambient and interior temperature of each collector and water temperature: which enters the solar collectors, at the 
exit of each collector and at the top of the storage tank. The efficiency on sunny day was $54.59 \%$, on combined day $48.32 \%$ and on cloudy day $30.01 \%$. The CPVC collector gained more temperature. A solar thermal plant with two flat collectors will produce hotter water, with coils of 10 turns of CPVC pipe of $1.5 \mathrm{~m} 2$ each and a thermally insulated $120 \mathrm{~L}$ water storage tank.

Keywords: Efficiency, Solar thermal.

\section{INTRODUCCIÓN}

La energía renovable es producida de manera natural, repetitiva y persistente en el medio ambiente en cualquier lugar de la Tierra; por ejemplo, la energía solar persiste y se repite día a día, varia de un lugar a otro y de una estación a otra. La energía renovable incluye la radiación solar, el viento, la biomasa (plantas cosechadas, estiércol), ríos (potencia hídrica), las mareas y calor geotérmico. También se le denomina energía verde o sustentable (Twidell y Weir, 2015). El Perú tiene un gran potencial de energías renovables, cuyo aprovechamiento reducirá el empleo de combustibles fósiles y la emisión de gases de efecto invernadero. Las tecnologías para su aplicación están disponibles y contribuirán a mejorar la calidad de vida de sus usuarios y la salud del planeta.

La energía contenida en la luz solar, llamada energía solar, puede convertirse directamente en calor, lo que se llama energía solar térmica; ejemplos son el calentamiento de agua fluyendo a través de un material absorbente negro que es calentado por la luz solar (terma solar) y el calentamiento de viviendas. La energía solar es radiación electromagnética que emite el sol. La cantidad real de radiación solar que llega a un lugar particular sobre la tierra es extremadamente variable. Además del diario regular y la variación anual debida al movimiento aparente del sol, las variaciones irregulares deben tomarse en cuenta pues son causadas por las condiciones atmosféricas locales, como las nubes. Esas condiciones influyen particularmente sobre los componentes directo y difuso de la radiación solar. La radiación solar directa es la parte de la luz solar que alcanza directamente una superficie. La dispersión de la luz solar en la atmósfera genera la radiación solar difusa. Una parte de la radiación solar que es reflejada por la superficie terrestre, llamada albedo, puede estar también presente en la radiación solar total (Jäger et al., 2014).

Vigo y Trigoso (2012), determinaron en la ciudad de Chachapoyas (2350 msnm y $17^{\circ} \mathrm{C}$ de temperatura ambiente) la eficiencia térmica de una terma solar con un colector de 1 $\mathrm{m}^{2}$ cuya parrilla de tubos se hizo de tubo de CPVC de $1 / 2$ " para agua caliente y accesorios del mismo material, y tanque de almacenamiento de agua de 100 litros aislado térmicamente. La temperatura del agua en el tanque de almacenamiento a las 16 horas y la eficiencia en días soleados fue de $44^{\circ} \mathrm{C}$ y $52 \%$, para días combinados fue de $36^{\circ} \mathrm{C}$ y $48 \%$ y para días nublados $25^{\circ} \mathrm{C}$ y $30 \%$; mientras que la temperatura interior del colector fue de 96 , 63 y $38^{\circ} \mathrm{C}$, respectivamente.

Para todos los tipos de días evaluados por Vigo y Trigoso (2012), con la terma solar se incrementó la temperatura del agua como mínimo $8,5^{\circ} \mathrm{C}$, con lo que el usuario dispone de agua a una temperatura adecuada para su higiene personal o lavado de utensilios de cocina, dándole una mejor calidad de vida. El agua almacenada a $44^{\circ} \mathrm{C}$ en el tanque con aislamiento térmico, durante la noche disminuyó su temperatura en $8^{\circ} \mathrm{C}\left(36^{\circ} \mathrm{C}\right)$; sin embargo, en la mañana siguiente se retiró 20 litros de agua y su temperatura solo disminuyó $1^{\circ} \mathrm{C}$ $\left(35^{\circ} \mathrm{C}\right)$, a pesar que la temperatura ambiente en horas de la noche en Chachapoyas era de 
$9^{\circ} \mathrm{C}$ y en la mañana era de $12^{\circ} \mathrm{C}$. Esto permite asegurar que la terma solar brindará confort al usuario y evitará que contraiga enfermedades respiratorias, por exponerse al agua fría debido a la baja temperatura ambiente.

Alrededor de la mitad de la energía consumida en el mundo es en forma de calor. Dos tercios de la demanda de calor es cubierta por carbón, petróleo y gas natural. El calor es mayormente usado en el sector industrial para facilitar los procesos químicos y en el sector residencial para calefacción y suministro de agua caliente. En un hogar promedio de Estados Unidos, la calefacción y el calentamiento de agua representan el $59 \%$ del consumo total de energía (Jäger et al., 2014).

La terma solar permite calentar agua únicamente con la radiación solar y almacenarla en su tanque aislado térmicamente para ser usada en cualquier momento. La eficiencia de la terma solar y su tiempo de vida dependen de los materiales empleados en su construcción. Consta de colector solar, tanque de almacenamiento de agua y sistema de tuberías.

El colector solar es una clase especial de intercambiador de calor que transforma la radiación solar en calor. En el colector solar, la transferencia de energía es desde una fuente distante de energía radiante (el sol) al fluido. El flujo de radiación incidente es aproximadamente $1100 \mathrm{~W} / \mathrm{m}^{2}$ (sin concentración óptica), y es variable. El colector plano puede diseñarse para aplicaciones que requieren suministro de energía a temperaturas moderadas, arriba de $100^{\circ} \mathrm{C}$ sobre la temperatura ambiente. Usan la radiación solar directa y la difusa, no requieren seguir la trayectoria del sol y necesitan poco mantenimiento. Su principal aplicación es para el calentamiento de agua empleando la radiación solar. La eficiencia del colector es influida por su calentamiento desde temprano en la mañana cuando está a baja temperatura hasta la temperatura final de operación en la tarde; también influye el comportamiento intermitente durante el día cuando las fuerzas impulsoras como la radiación solar y el viento cambian rápidamente (Duffie \& Beckman, 2013).

El sistema de tuberías tiene la función de transportar el agua fría o caliente a través de los elementos que forman la terma solar. Se puede utilizar tuberías de cobre, fierro galvanizado o de policloruro de vinilo especial para agua caliente (CPVC). Debe estar apropiadamente aislado, tanto para su protección como para evitar las pérdidas de calor del agua.

La eficiencia energética $\left(\eta_{E}\right)$ de una terma solar se define como el cociente entre la energía térmica ganada por la terma y la energía solar recibida o disponible ese día. Se debe tener en cuenta la evolución de la temperatura en el tanque de agua y la radiación solar correspondiente.

La terma solar basa su operación en los siguientes principios: (Valera, 2007; Duffie \& Beckman, 2013; Jäger et al., 2014)

- La pintura esmalte negro mate absorbe al máximo la radiación directa y la difusa. El coeficiente de absorción a es la fracción de radiación que absorbe; pintura blanca $a \approx 0,25$, pintura negra esmalte brillante $a \approx 0,80$ y pintura esmalte negro mate $a$ $\approx 0,90$.

- El efecto invernadero generado por el vidrio que es transparente a la radiación solar (visible e infrarroja); pero opaco a la radiación infrarroja lejana que emite el cuerpo calentado, actuando como una trampa de calor.

- La conducción del calor por un gradiente de temperatura, ocurre entre la lámina metálica absorbente y los tubos, y de éstos al agua que circula por su interior.

- El agua circula en el sistema por termosifón producido por el incremento de su temperatura en el colector solar. El agua caliente es menos densa que la fría y sube 
al tanque, estableciéndose una circulación natural, sin necesidad de equipo de bombeo.

Con la presente investigación se ha validado una propuesta amigable con el medio ambiente constituida por un prototipo de terma solar para mejorar la calidad de vida de los pobladores de zonas altoandinas, sobre todo en temporadas de heladas y friaje; épocas en que el confort térmico del agua de la terma solar los salvaguardará de enfermedades recurrentes como la neumonía. El uso de la terma solar permitirá que cada familia de cinco integrantes disponga de agua caliente para la higiene personal, de manera que sus hijos se prevengan de enfermedades respiratorias, digestivas y cutáneas; eviten el contacto con el agua a baja temperatura y vayan mejor presentados a su institución educativa.

\section{MATERIALES Y MÉTODOS}

Se construyó una terma solar con dos colectores, cada uno de 1,5 m de largo por $1 \mathrm{~m}$ de ancho y 0,10 $\mathrm{m}$ de altura; cuya caja se hizo de triplay de 0,01 m de espesor, con el fondo y los lados aislados térmicamente con plancha de tecnopor de 0,0254 m (1") de espesor, cubierta con papel de aluminio. Para un colector se hizo el serpentín con tubos y accesorios de cobre de 0,0127 m (1/2") (Figura 1), y para el otro colector el serpentín se hizo con tubos y accesorios de CPVC de 0,0127 m (1/2"), cada serpentín tiene 10 curvas en U (Figura 1), se fijó firmemente sobre una plancha delgada de fierro galvanizado y ambos componentes se pintaron con esmalte negro mate para maximizar la absorción de calor de la radiación solar, luego se colocaron cada uno dentro de su caja de triplay que se tapó con plancha de vidrio incoloro de 0,004 m de espesor para generar efecto invernadero. Los colectores se ubicaron en la dirección norte-sur, con el lado sur levantado $30^{\circ}$ para compensar la curvatura terrestre, conectados en paralelo, es decir, el agua ingresa en la base de ambos en simultáneo, los mismos que calentaron el agua con la radiación solar. El agua caliente circuló por termosifón natural hacia el tercio superior del tanque de almacenamiento de agua de 120 litros, de polietileno de alta densidad, ubicado en el lado sur de los colectores para que no les haga sombra, aislado térmicamente con tecnopor y colocado dentro de una caja de triplay de 0,01 $\mathrm{m}$ de espesor, con cobertura interior de 0,0508 m $\left(2^{\prime \prime}\right)$ de espesor de tecnopor, protegida de las Iluvias con techo de calamina a dos aguas. El tanque de almacenamiento y los colectores se conectaron con tubos y accesorios de PVC pesado de 0,0127 m (1/2") de diámetro, aislados térmicamente con bloques de tecnopor de 1,3 $\mathrm{m}$ de largo por 0,10 $\mathrm{m}$ por 0,15 m (Figura 2).
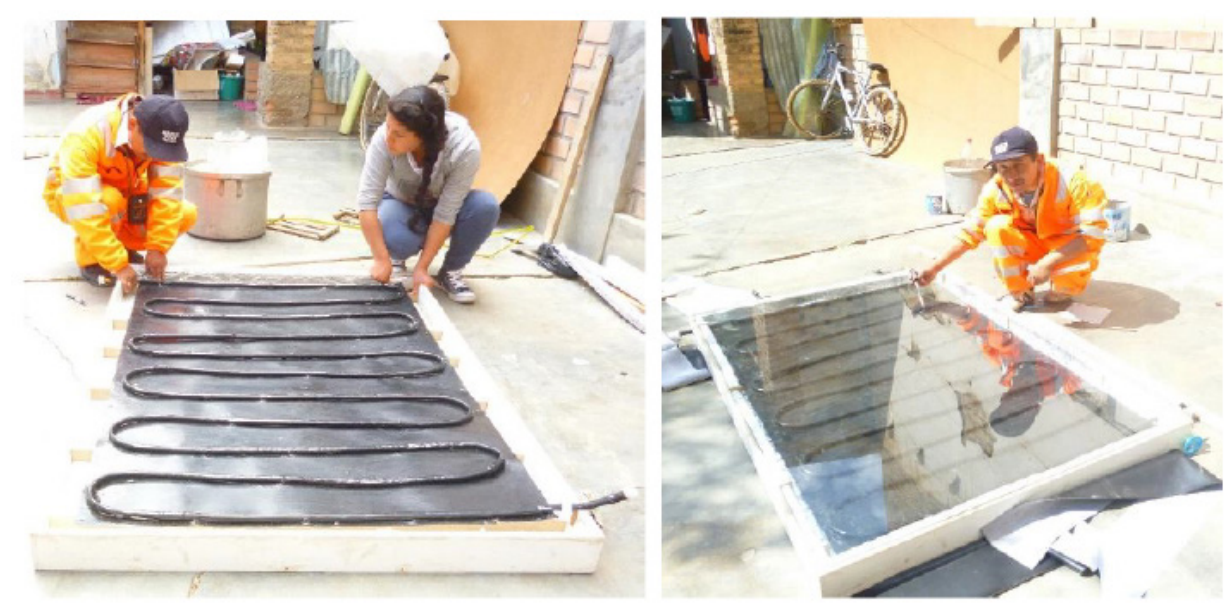

Figura 1. Ensamble del colector de tubería de cobre. 

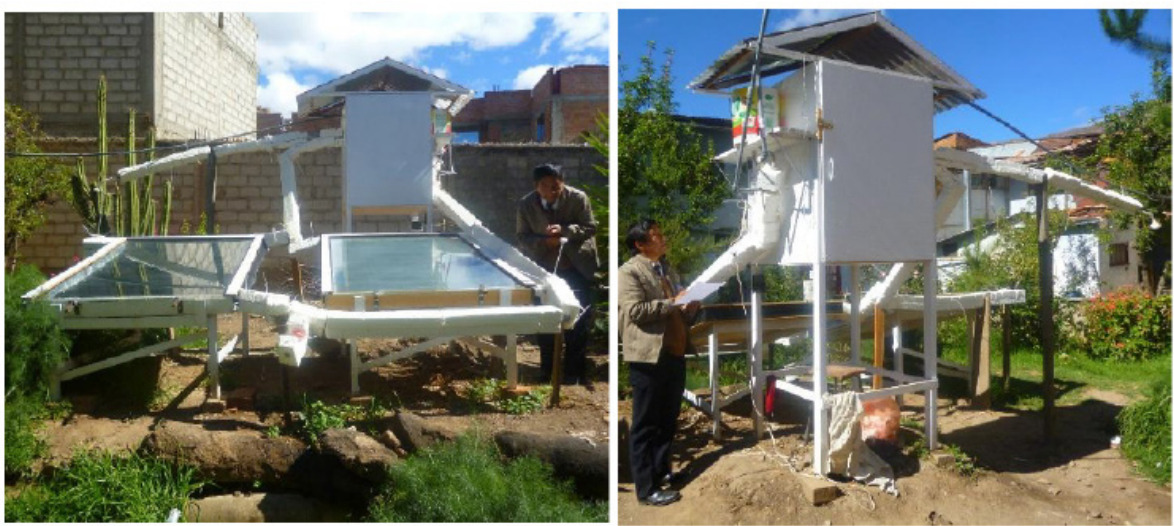

Figura 2. Terma solar con dos colectores en paralelo evaluada en la presente investigación.

La terma solar se construyó, instaló y evaluó en la vivienda ubicada en el Jr. Malecón Gálvez N 528, Tarma, Región Junín - Perú; a 3050 msnm; con coordenadas 18L 0424625 -UTM 8737361 con Latitud $11^{\circ} 25^{\prime} 16.21^{\circ} \mathrm{S}$ - Longitud $75^{\circ} 41^{\prime} 27.44^{\circ} \mathrm{O}$.

Para determinar la eficiencia de la terma solar, se tomaron datos en tres tipos de días: soleados (presencia visible del sol todo el día), combinados (presencia visible parcial del sol por presencia de nubes) y nublados (no hay presencia visible del sol, todo el día esta nublado). Cada media hora desde las 8:00 hasta las 16:00 horas, se midió y registró las siguientes temperaturas: del agua que entra a los colectores solares, del interior de cada colector, del agua a la salida de cada colector y del agua en la parte superior del tanque de almacenamiento. En simultáneo, se midió y registró la radiación solar con un piranómetro (digitale multimeter mit solarmeetfunctie) marca VoltCraft SPM-1 DMM y con un termohigrómetro digital se midió la temperatura ambiente.

La eficiencia de la terma solar, para cada tipo de día evaluado, se calculó con la ecuación (Valera, 2007; Duffie \& Beckman, 2013; Jäger et al., 2014; Kyoung-ho Leea \& Nam-choon Baekb, 2014; Weiss, 2016):

$\eta_{\mathrm{E}}=$ Energía térmica útil o ganada/ energía solar recibida
Energía térmica ganada: $E_{T}=m . c . \Delta T$

Donde:

m: masa de agua en la terma $=100 \mathrm{~L}=1 \times 10^{5}$

$\mathrm{g}$

c: calor específico del agua $=4,18 \mathrm{~J} / \mathrm{g} \cdot{ }^{\circ} \mathrm{C}$

$\Delta T=$ Temperatura máxima del agua (16:00 horas) - Temperatura del agua en el tanque al inicio (8:00 horas), ${ }^{\circ} \mathrm{C}$.

Energía solar recibida: $E_{S}=e_{s}$. $A$

Donde:

es: Energía solar recibida, es el área bajo la curva de radiación solar vs tiempo, calculada con el método del área de los rectángulos bajo esa curva.

A: Área del colector solar, m².

\section{RESULTADOS}

Se registraron los datos experimentales durante 35 días, en los que se tuvo los tres tipos de días propuestos para la evaluación: soleados (Figura 3 y 4), combinados (Figura 5 y 6) y nublados (Figura 7 y 8 ). En cada uno de los casos, el calor del sol calentó los colectores y éstos transfirieron su calor al agua contenida en su serpentín ocasionando que disminuya su densidad, generando un termosifón natural que hizo que el agua fluya hacia la parte superior de los tubos y de allí al tercio superior del tanque de almacenamiento, lo que a su vez originó que el agua fría de la base del tanque de almacenamiento entre a la base 
de cada colector y se repita este ciclo de manera continua, hasta que la temperatura del agua dentro de los dos colectores sea igual a la temperatura del agua dentro del tanque de almacenamiento.

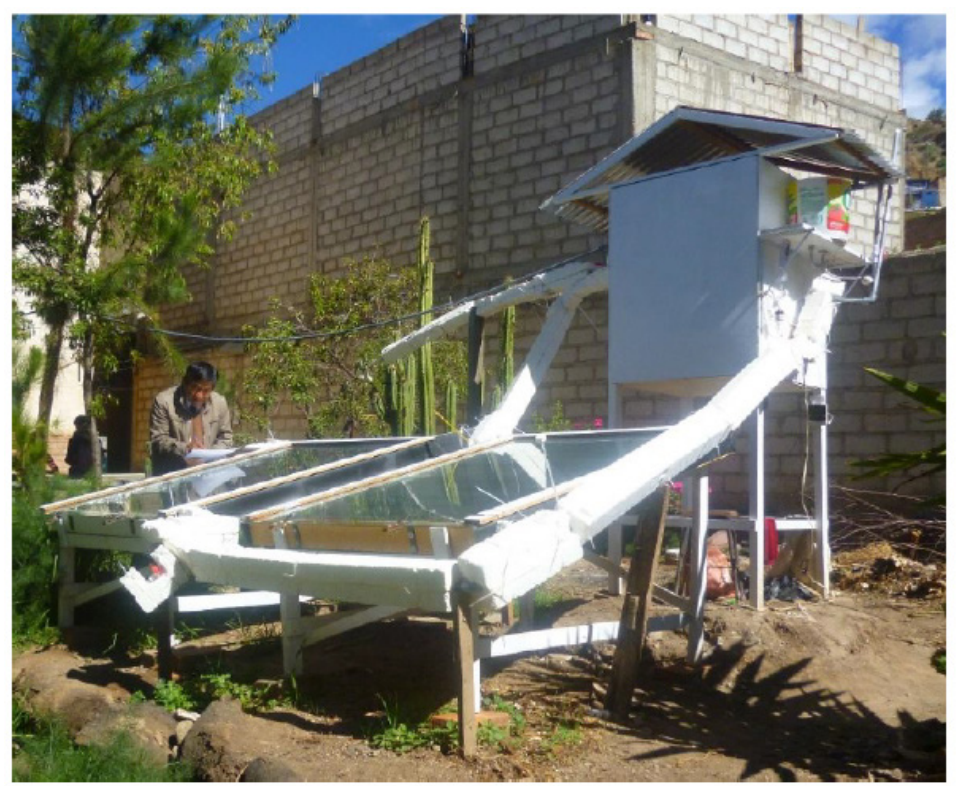

Figura 3. Toma de datos en la terma solar en día soleado.

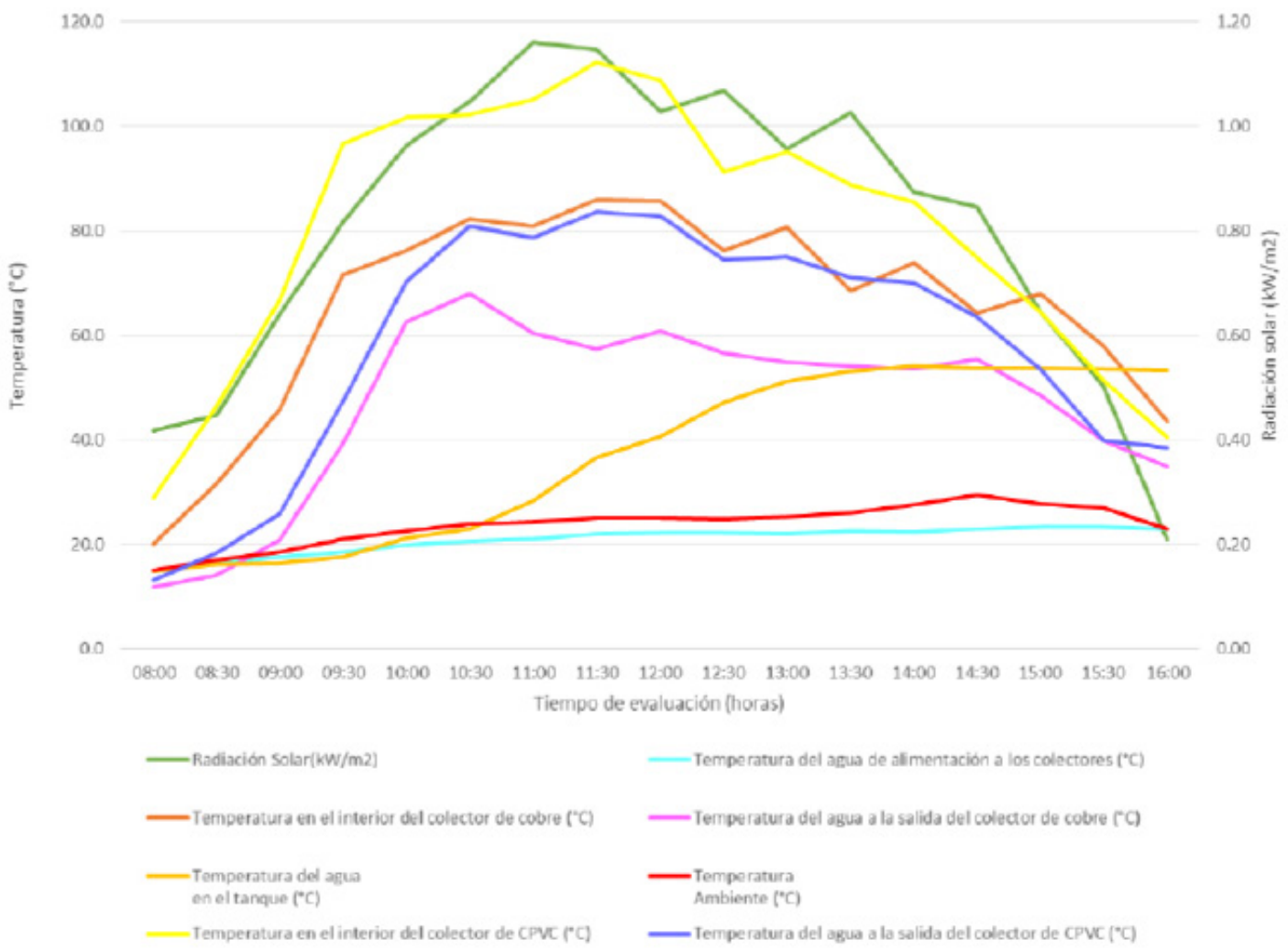

Figura 4. Temperaturas y radiación solar en función al tiempo de evaluación de la terma solar en días soleados. 
En días soleados (Figura 4), la radiación solar se mantuvo sobre 1,00 kW/m² desde las 10:00 am hasta las 2:15 pm, y presentó un pico de 1,161 kW/m² a las 11:00 am. La temperatura interior del colector de CPVC estuvo sobre los $80^{\circ} \mathrm{C}$ desde las 9:00 am hasta las 2:30 pm, tuvo un máximo de $112^{\circ} \mathrm{C}$ a las $11: 30 \mathrm{am}$; mientras que el colector de cobre sólo superó los $80^{\circ} \mathrm{C}$ desde las $10: 15 \mathrm{am}$ hasta la 1:00 pm, y alcanzó una temperatura máxima de $85,8^{\circ} \mathrm{C}$ a las 11:30 am. La temperatura del agua a la salida del colector de CPVC fue en promedio $20^{\circ} \mathrm{C}$ más que del colector de cobre. La temperatura del agua en el tanque de almacenamiento se incrementó constantemente desde $14,9^{\circ} \mathrm{C}$ a las $8: 00$ am hasta $53,4^{\circ} \mathrm{C}$ a la $1: 30 \mathrm{pm}$, la que se mantuvo hasta las 4:00 pm (fin del registro de datos); mientras que la temperatura ambiente varió desde los $15,1^{\circ} \mathrm{C}$ a las $8: 00 \mathrm{am}$ hasta los $29,4^{\circ} \mathrm{C}$ a las $2: 30 \mathrm{pm}$ y luego disminuyó a $22,9^{\circ} \mathrm{C}$ a las $4: 00 \mathrm{pm}$.

Para calcular la eficiencia de la terma solar en días soleados, se aplicó la ecuación (2):

$$
\begin{aligned}
& \mathrm{E}_{\mathrm{T}}=\mathrm{m} . \mathrm{c} \cdot \Delta \mathrm{T} \\
& \mathrm{E}_{\mathrm{T}}=\left(1,2 \times 10^{5} \mathrm{~g}\right) \times\left(4,18 \mathrm{~J} / \mathrm{g} \cdot{ }^{\circ} \mathrm{C}\right) \times\left(54,1^{\circ} \mathrm{C}-14,9^{\circ} \mathrm{C}\right) \\
& \mathrm{E}_{\mathrm{T}}=1,966272 \times 10^{7} \mathrm{~J}
\end{aligned}
$$

La energía solar recibida se calculó con la ecuación (3):

$\mathrm{E}_{\mathrm{s}}=$ es. $\mathrm{A}$

El área de cada colector solar fue de 1,5 $\mathrm{m}^{2}$ y como ambos están conectados en paralelo, el área se considera como $1,5 \mathrm{~m}^{2}$. El área bajo la curva de radiación solar vs tiempo fue:

$$
\begin{aligned}
& \text { es }=6,67 \mathrm{kWh} / \mathrm{m} 2 \\
& \text { es }=\left(6,67 \mathrm{kWh} / \mathrm{m}^{2}\right) \times \\
& \text { es }=2,4012 \times 10^{7} \mathrm{~J} / \mathrm{m}^{2} \\
& \text { Es }=2,4012 \times 10^{7} \mathrm{~J} / \mathrm{m}^{2} \times 1,5 \mathrm{~m}^{2} \\
& \text { Es }=3,6018 \times 10^{7} \mathrm{~J}
\end{aligned}
$$

La eficiencia de la terma solar se calculó con la ecuación (1):

$\eta \mathrm{E}=$ Energía térmica útil o ganada/ energía solar recibida

$\eta \mathrm{E}=$

$\eta E=0,5459$; en porcentaje la eficiencia será de 54,59\% para días soleados.

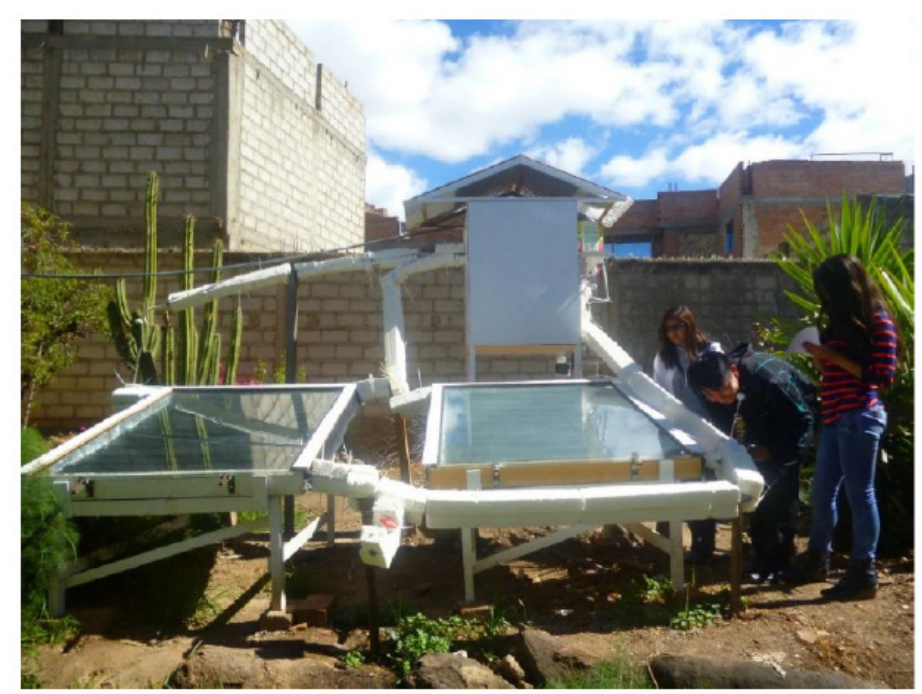

Figura 5. Toma de datos en la terma solar en día combinado. 


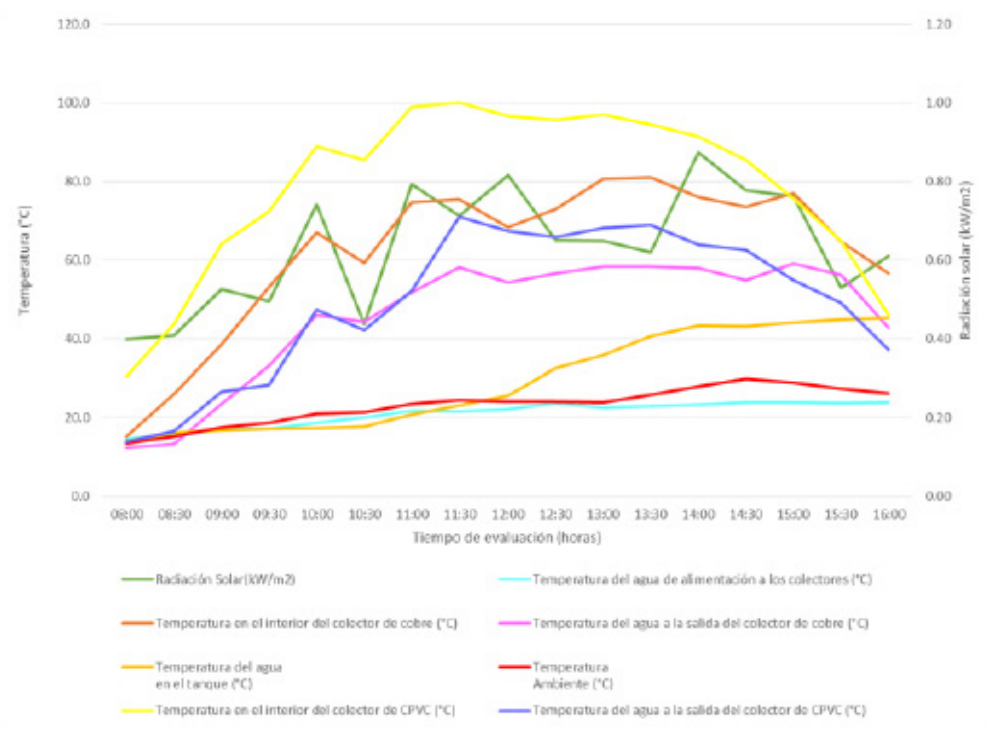

Figura 6. Temperaturas y radiación solar en función al tiempo de evaluación de la terma solar en días combinados.

En días combinados (Figura 6), se presentaron picos de radiación solar con un valor promedio de $0,80 \mathrm{~kW} / \mathrm{m}^{2}$ a las 11:00 am y 12:00 m y un último de $0,88 \mathrm{~kW} / \mathrm{m}^{2}$ a las $2: 00 \mathrm{pm}$, los demás valores de radiación solar fueron menores a $0,80 \mathrm{~kW} / \mathrm{m}^{2}$. La temperatura interior del colector de CPVC llegó a $100^{\circ} \mathrm{C}$ a las 11:30 am y se mantuvo alrededor de $90^{\circ} \mathrm{C}$ hasta las 2:00 pm y luego bajó rápidamente hasta $41^{\circ} \mathrm{C}$; mientras que el colector de cobre llegó a $80^{\circ} \mathrm{C}$ a la 1:00 pm; a partir de las $3: 00 \mathrm{pm}$ la temperatura de ambos colectores fue de $78^{\circ} \mathrm{C}$ y bajaron hasta $45^{\circ} \mathrm{C}$. La temperatura del agua a la salida de ambos colectores fue similar desde las 8:00 am hasta las 11:00 am, luego fue mayor en $10^{\circ} \mathrm{C}$ aproximadamente la temperatura de salida del agua del colector de CPVC, hasta las 3:00 pm en que volvieron a coincidir en valores y tendencia a disminuir. La temperatura del agua en el tanque de almacenamiento se incrementó constantemente hasta superar los $40^{\circ} \mathrm{C}$ a partir de la 1:30 pm; mientras que la temperatura ambiente se mantuvo alrededor de los $20^{\circ} \mathrm{C}$. La eficiencia de la terma solar en día combinado fue de 48,32\%.

En días nublados (Figura 8), la radiación solar tuvo varios picos con un promedio de 0,45 kW/ $\mathrm{m}^{2}$ en todo el día. El pico de radiación de $0,70 \mathrm{~kW} / \mathrm{m}^{2}$ a las 10:00 am, originó una diferencia de temperatura entre ambos colectores de $15^{\circ} \mathrm{C}$ en promedio, siendo mayor la temperatura interior del colector de CPVC, hasta que sus valores de temperatura y su tendencia fueron iguales a partir de las 12:30 pm. La temperatura del agua en la salida de ambos colectores tuvo tendencia similar, con un promedio de $40^{\circ} \mathrm{C}$. La temperatura del agua en el tanque aumentó desde $16^{\circ} \mathrm{C}$ a las 8:00 am, superó los $20^{\circ} \mathrm{C}$ a partir de las $11: 30 \mathrm{am}$, alcanzando los $28^{\circ} \mathrm{C}$ a las $2: 30 \mathrm{pm}$; mientras que la temperatura ambiente estuvo entre $13,5^{\circ} \mathrm{C}$ y un promedio de $20^{\circ} \mathrm{C}$ en ese mismo rango de tiempo. La eficiencia de la terma solar en día nublado fue de 30,01\%. 


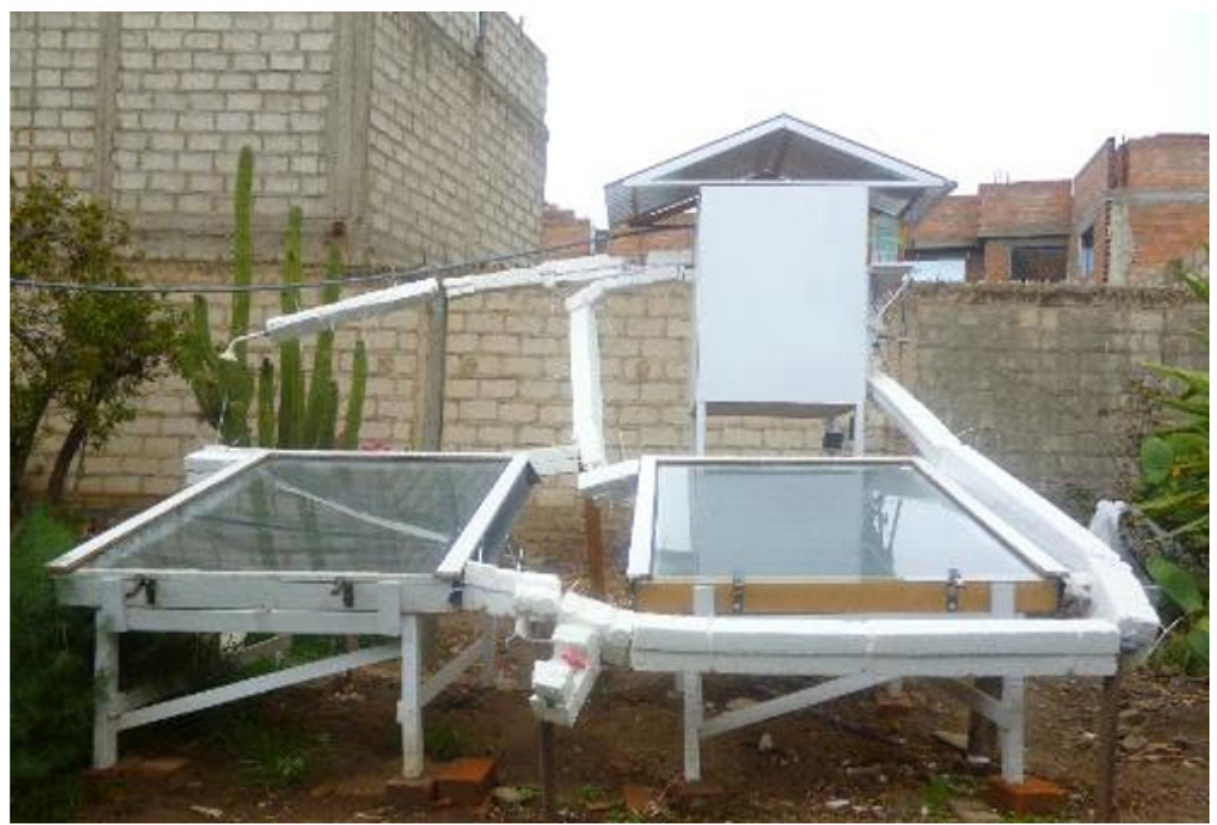

Figura 7. Toma de datos en la terma solar en día nublado.

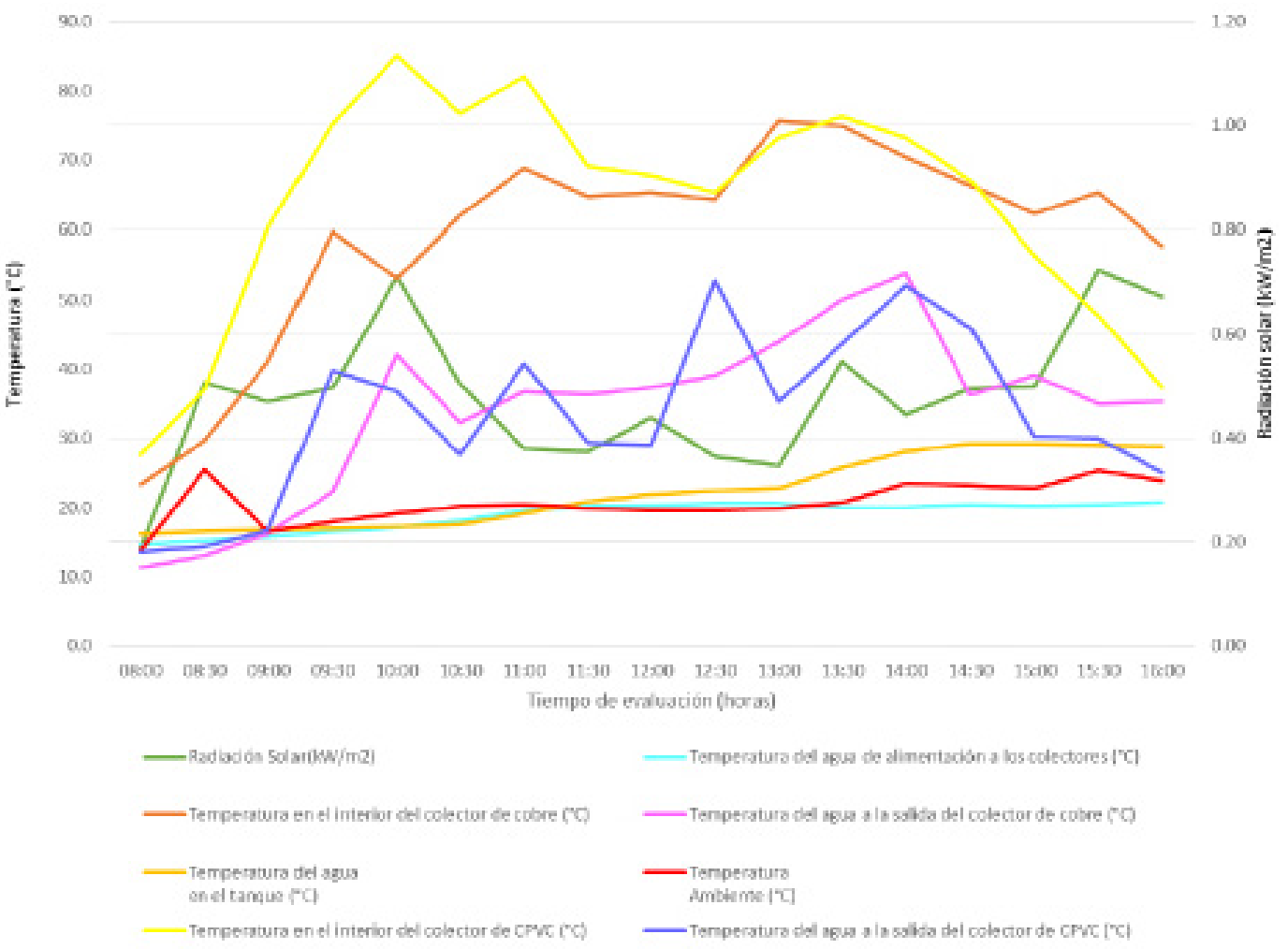

Figura 8. Temperaturas y radiación solar en función al tiempo de evaluación de la terma solar en días nublados. 


\section{DISCUSIÓN}

En la presente investigación se determinó la eficiencia térmica de una terma solar para calentar agua con un colector solar plano de CPVC y otro similar de cobre, cada uno de 1,5 $\mathrm{m}^{2}$ conectados en paralelo, con un tanque de almacenamiento de agua caliente de 120 $L$ aislado térmicamente. Se midieron y se registraron los datos en tres tipos de días: soleados, combinados y nublados; habiendo sido la ganancia de temperatura de 39,2, 27 y $12,6^{\circ} \mathrm{C}$, respectivamente, desde las 8:00 am en que el agua tenía una temperatura de $14^{\circ} \mathrm{C}$ en promedio hasta las 4:00 pm; cada día se evaluó la terma solar con agua recién abastecida de la red de agua potable. El incremento de temperatura del agua estuvo relacionado directamente con la presencia visible del sol y la ganancia de temperatura asegura que aunque el día esté nublado, la terma solar abastecerá con agua por lo menos $10^{\circ} \mathrm{C}$ más que la temperatura del agua de la red de abastecimiento, para confort de los usuarios.

En la ciudad de Tarma, Región Junín, Perú; la radiación solar en día soleado superó los 1000 $\mathrm{W} / \mathrm{m}^{2}$, desde las 10:00 am hasta la 1:30 pm, debido a su clima seco por la escasa vegetación. El aumento y la disminución de la temperatura del interior de los colectores solares evaluados tiene relación directa con la radiación solar. Cuando la radiación solar superó los $0,8 \mathrm{~kW} / \mathrm{m}^{2}$, el colector hecho con tubos y accesorios de CPVC ganó más temperatura que el colector hecho con tubos y accesorios de cobre de iguales dimensiones, con una diferencia promedio de $20^{\circ} \mathrm{C}$.

En días combinados, hubo tres picos de radiación solar con un valor promedio de $0,80 \mathrm{~kW} / \mathrm{m}^{2}$ a las 11:00 am y 12:00 m y un último de 0,88 kW/ $\mathrm{m}^{2}$ a las 2:00 pm, los demás valores de radiación solar fueron menores a $0,80 \mathrm{~kW} / \mathrm{m}^{2}$. Sólo cuando la radiación solar superó los 0,60kW/m², la diferencia de temperatura entre ambos colectores fue de $10^{\circ} \mathrm{C}$ en promedio, siendo mayor la temperatura del colector de CPVC.
En días nublados, la radiación solar tuvo valores menores a 0,60 kW/ $/ \mathrm{m}^{2}$ por lo que no se presentó diferencia significativa en la temperatura de ambos colectores solares; a diferencia de lo ocurrido en días soleados y combinados.

En días soleados, el colector de CPVC Ilegó a un máximo de $112^{\circ} \mathrm{C}$ y el de cobre a $80^{\circ} \mathrm{C}$; en día combinado fue de 100 y $80^{\circ} \mathrm{C}$ y en día nublado de 85 y $75^{\circ} \mathrm{C}$, respectivamente. Lo obtenido con el colector de cobre está de acuerdo con lo mencionado por Valera (2007), que un colector plano puede llegar a temperaturas entre $65-90^{\circ} \mathrm{C}$, mientras que lo obtenido con el colector de CPVC supera ese rango.

A pesar que el plástico tiene una conductividad térmica menor, el colector de CPVC ganó más temperatura y en consecuencia calentó más el agua que el colector de cobre de iguales dimensiones. La diferencia de temperatura del interior de ambos fue en promedio de $20^{\circ} \mathrm{C}$, igual entre las temperaturas del agua a la salida de ellos. De manera general, el colector de CPVC se calentó más con la radiación solar y transfirió su calor al agua de manera más efectiva, demostrándose con ello que una terma solar con dos colectores de este mismo material tendrá una eficiencia mayor porque producirá una mayor diferencia de temperatura del agua, teniendo en cuenta que, según la ecuación (2), a mayor diferencia de temperatura, mayor eficiencia de la terma solar.

En Tarma, en día soleado, la energía solar recibida fue de $6,67 \mathrm{kWh} / \mathrm{m}^{2}$, en día combinado fue de $5,19 \mathrm{kWh} / \mathrm{m}^{2}$, y en día nublado fue de 3,9 kWh/m². Vigo y Trigoso (2012), determinaron en Chachapoyas (ceja de selva a $2350 \mathrm{msnm}) 5,42,4,01$ y $3,22 \mathrm{kWh} / \mathrm{m}^{2}$ para los mismos tipos de días. La radiación solar en la ciudad de Cajamarca (2700 msnm) es de 4,68 kWh/m², valor que está entre la radiación solar de días soleados y combinados de 
Chachapoyas, mientras que en Huancayo es de 5,98 kWh/m² que según Valera (2007), es la zona con mayor insolación de la sierra peruana; sin embargo, en Tarma fue mayor en día soleado quizá a consecuencia del cambio climático que se viene acentuando con el paso de los años.

La eficiencia de la terma solar evaluada en Tarma fue en día soleado de 54,59\%, en día combinado fue de $48,32 \%$ y en día nublado fue de 30,01\%; valores ligeramente mayores a los reportados por Vigo y Trigoso (2012), en Chachapoyas de 52,48 y $30 \%$, respectivamente. La tendencia decreciente de la eficiencia se debió directamente a la disminución de la presencia visible del sol.

Después de un día soleado en Tarma, dejando el agua caliente en el tanque de almacenamiento desde las 4:00 pm $\left(54,1^{\circ} \mathrm{C}\right)$ hasta las 6:30 am del día siguiente, se encontró que el agua baja en un promedio de $11,9^{\circ} \mathrm{C}$ en ese tiempo. Al llenar, a través de la ducha de la terma solar, un primer balde de 20 litros (suficiente para el baño de una persona), la temperatura es la misma y al retirar de 2 a 4 baldes, la temperatura disminuye en $1^{\circ} \mathrm{C}$, con lo cual el agua aún está sobre los $40^{\circ} \mathrm{C}$ en el tanque de almacenamiento. Esto permite asegurar que la terma o calentador solar de agua brindará confort a los usuarios y evitará que contraigan enfermedades respiratorias, por exponerse al agua fría debido a la temperatura ambiente baja.

La terma solar requiere escaso mantenimiento para seguir funcionando, solo es necesario una limpieza semanal del vidrio que cubre cada uno de los colectores solares para dejar paso libre a los rayos solares y conservar su eficiencia. La energía solar es segura para su uso y su abastecimiento durante todo el año, es ecológica y amigable con el medio ambiente. Es una solución económica para abastecer a la vivienda con agua caliente y su costo se puede financiar con el ahorro del gasto en electricidad o gas para este mismo propósito.

\section{CONCLUSIONES}

- Se midieron y se registraron los datos en tres tipos de días: soleados, combinados y nublados; habiendo sido la ganancia de temperatura de $39,2,27$ y $12,6^{\circ} \mathrm{C}$, respectivamente, desde las 8:00 am en que el agua tenía una temperatura de $14^{\circ} \mathrm{C}$ en promedio hasta las 4:00 pm; cada día se evaluó la terma solar con agua recién abastecida de la red de agua potable.

- El incremento de temperatura del agua estuvo relacionado directamente con la presencia visible del sol y la ganancia de temperatura asegura que aunque el día esté nublado, la terma solar abastecerá con agua por lo menos $10^{\circ} \mathrm{C}$ más que la temperatura del agua de la red de abastecimiento para confort de los usuarios.

- El colector hecho con tubos y accesorios de CPVC ganó más temperatura y en consecuencia calentó más el agua que el colector hecho con tubos y accesorios de cobre, sobre todo cuando la radiación solar superó los 0,60 kW/m2.

- Una terma solar con dos colectores de CPVC tendrá una eficiencia mayor porque producirá una mayor diferencia de temperatura del agua, teniendo en cuenta que, a mayor diferencia de temperatura, mayor eficiencia de la terma solar.

- La eficiencia térmica de una terma solar evaluada en Tarma para calentar agua con un colector solar plano de CPVC y otro similar de cobre, cada uno de 1,5 m2 conectados en paralelo, con un tanque de almacenamiento de agua caliente de 120 $\mathrm{L}$ aislado térmicamente, fue en día soleado de $54,59 \%$, en día combinado de 48,32 $\%$ y en día nublado fue de $30,01 \%$.

- Una terma solar con dos colectores planos, con serpentín de 10 vueltas hecho con tubos y accesorios de CPVC de 1,5 m2 cada uno y tanque de almacenamiento de agua de 120 litros aislado térmicamente, es el modelo adecuado para instalar en viviendas en zonas altoandinas. 


\section{REFERENCIAS BIBLIOGRÁFICAS}

Duffie, John A. \& William A. Beckman. (2013). Solar engineering of thermal processes. Fourth Edition. John Wiley \& Sons, Inc. New Jersey, USA.

Jäger, Klaus, Olindo Isabella, Arno H.M. Smets, René A.C.M.M. van Swaaij \& Miro Zeman. 2014. Solar energy Fundamentals, technology and systems. Delft University of Technology. Netherland.

Kyoung-ho Leea \& Nam-choon Baekb. (2014). A modified efficiency equation of solar collectors. SHC 2013, International Conference on Solar Heating and Cooling for Buildings and Industry. September 23-25, 2013, Freiburg, Germany. Energy Procedia 48 (2014) 145 - 149.

Twidell, John \& Tony Weir. (2015). Renewable energy resources. Third edition. Routledge, Taylor and Francis Group. London and New York.

Valera Palacios, A. 2007. Energía solar II. Edición a cargo de la Asamblea Nacional de Rectores. Lima - Perú.

Vigo Cruz, Lady V. y Ronal R. Trigoso Valle. (2012). Eficiencia térmica de un calentador solar de agua de 80 litros con colector de 1 $\mathrm{m}^{2}$. Tesis para obtener el título profesional de Ingeniero Agroindustrial. Universidad Nacional Toribio Rodríguez de Mendoza de Amazonas. Perú.

Weiss, Werner. (2016) Design of solar thermal systems - Calculation methods. AEE - Institute for Sustainable Technologies. Austrian Development Cooperation. www.aee-intec.at.

\section{CORRESPONDENCIA:}

Dr. Miguel Barrena Gurbillón

miguel.barrena@untrm.edu.pe 